\title{
Erkenntnisse und Konzepte zur langfristigen Entwicklung der Landwirtschaft im Alpenraum
}

\section{Working Paper}

\section{Author(s):}

Rieder, Peter

Publication date:

1996

Permanent link:

https://doi.org/10.3929/ethz-a-004996388

Rights / license:

In Copyright - Non-Commercial Use Permitted

Originally published in:

Schriftenreihe / ETH Zürich, Institut für Agrarwirtschaft 1996(3) 
ETH Zürich

Institut für Agrarwirtschaft/Institut d'économie rurale

\section{Schriftenreihe Publications}

$1996 / 3$

ERKENNTNISSE UND KONZEPTE ZUR LANGFRISTIGEN ENTWICKLUNG DER LANDWIRTSCHAFT IM ALPENRAUM

Peter Rieder

Dezember 1996

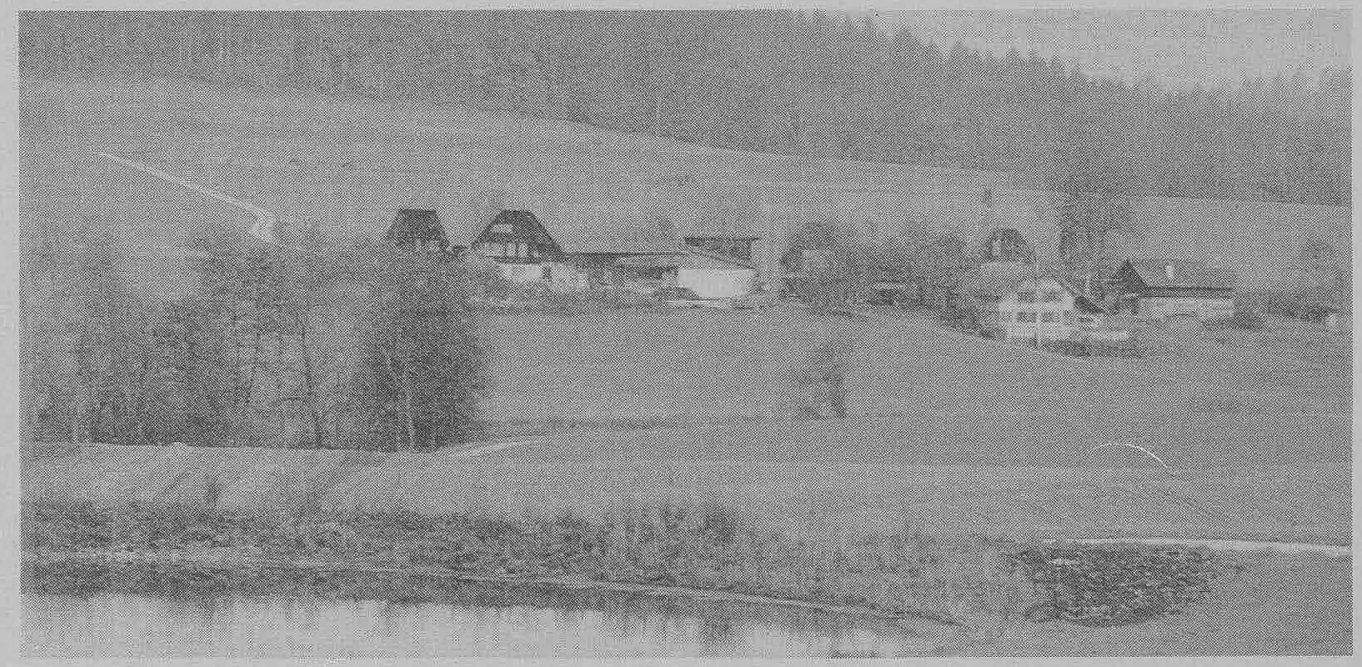

ETH-Zentrum, 8092 Zürich 
$1996 / 3$

ERKENNTNISSE UND KONZEPTE ZUR LANGFRISTIGEN ENTWICKLUNG DER LANDWIRTSCHAFT IM ALPENRAUM

Peter Rieder

Dezember 1996

Adresse des Autors:

Institut für Agrarwirtschaft

ETH-Zentrum

8092 Zürich

Erste Auflage (50 Exemplare) 


\section{Erkenntnisse und Konzepte zur langfristigen Entwicklung der Landwirtschaft im Alpenraum}

\section{Einleitung}

Weltweite Veränderungen wie Liberalisierungen der Agrarmärkte, aber auch Deregulierungen der nationalen Marktordnungen werfen Fragen nach deren langfristigen Auswirkungen auf die Landwirtschaft im Alpenraum auf. Denn bekanntlich weist die Landwirtschaft des Alpenraumes absolute Kostennachteile gegenüber anderen Regionen auf. In diesem Artikel werden diese Fragen in vier Kapiteln angegangen. Zuerst folgen Ausführungen zum Strukturwandel in der Landwirtschaft der Schweiz als Ganzes. Im zweiten Kapitel werden die regionalen Auswirkungen der eingeleiteten Reform der Agrarpolitik (AP 2002) besprochen. Anschliessend wird das Leitbild der Ökologischen Wettbewerbslandwirtschaft beschrieben und gefragt, wieweit dieses auch seine Gültigkeit für den Alpenraum besitzt. Abschliessend werden in einem vierten Teil die Probleme aufgezeigt, die bei der Umsetzung von Leitbildern mit Verteilungseffekten, öffentlichen Gütern und externen Effekten bestehen.

\section{Strukturwandel als kaum beeinflussbare Grundströmung}

\section{Komponenten der landwirtschaftlichen Strukturen}

Um landwirtschaftliche Strukturen zu beschreiben, verwendet man Angaben zur landwirtschaftlich genutzten Fläche, zur Anzahl Arbeitskräfte und zum Realkapital (Gebäude, Maschinen und Einrichtungen). Diese drei Strukturkomponenten sind für jedermann sichtbar und werden zudem bei Betriebszählungen statistisch erfasst. Die Gesamtheit all dieser Faktoren auf allen Betrieben ergibt die Agrarstruktur eines Landes. Nicht unter den Strukturbegriff fallen Flussgrössen wie Erträge, Erlöse und Einkommen.

In Abbildung 1 ist ein Strukturbild dargestellt, das die absolute Zahl der Betriebe (rechte Skala) und die prozentualen Anteile pro Grössenklasse (linke Skala) im Zeitablauf enthält. In Tabelle 1 sind die absoluten und relativen Veränderungen der Produktionsfaktoren Arbeitskräfte, Realkapital und Fläche der letzten vierzig Jahre aufgeführt. Alle diese Angaben brauchen wir, um den Strukturwandel zu erklären und um daraus Lehren für den zu erwartenden zukünftigen Strukturwandel zu ziehen. 


\section{Abb. 1: Prozentuale Verteilung nach Betriebsgrössen und die Entwick- lung der Betriebszahl}

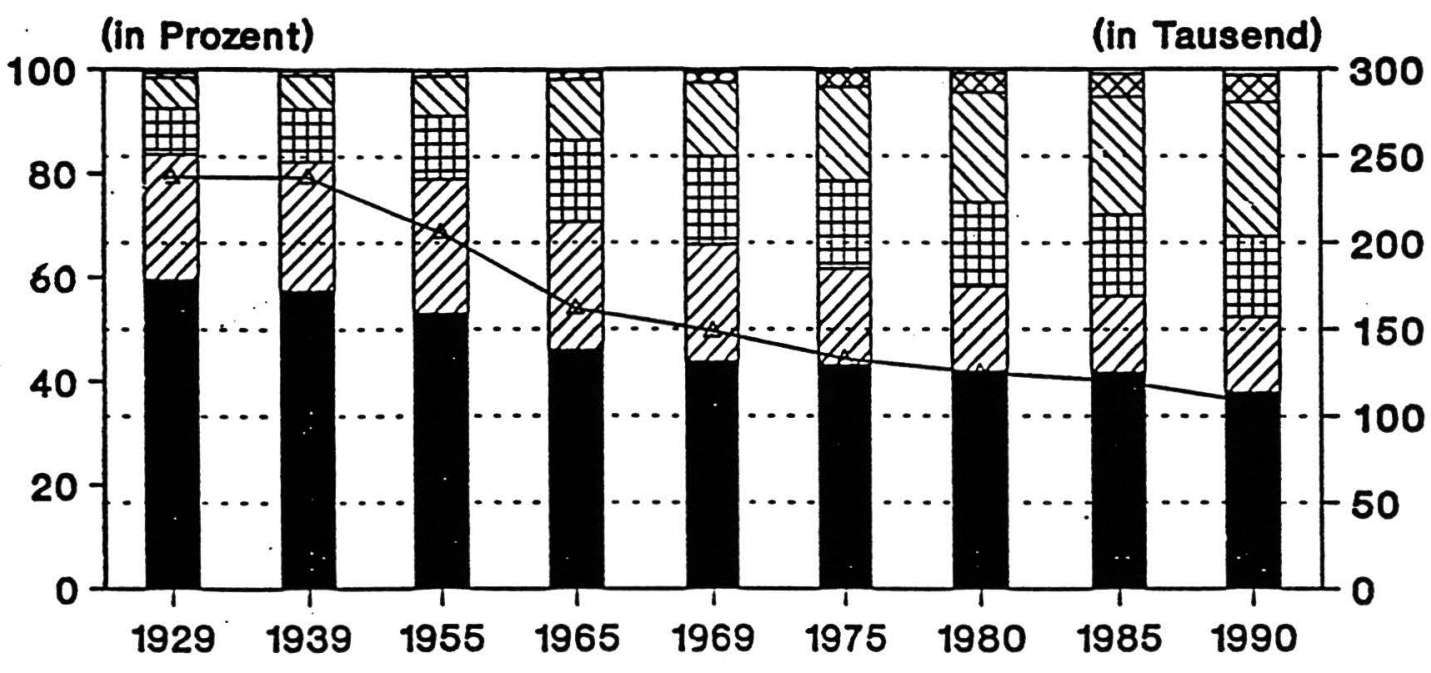

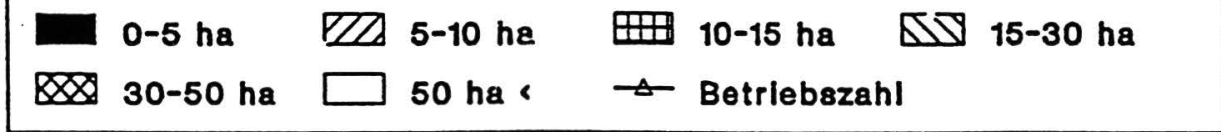

Quelle: SBS, Statistische Erhebungen und Schätzungen, div. Jg., BZ 1990

Tab. 1: Entwicklung der drei Produktionsfaktoren seit 1955

\begin{tabular}{|c|c|c|c|c|c|c|}
\hline Jahr & \multicolumn{2}{|c|}{$\begin{array}{c}\text { Arbeitskräfte männ- } \\
\text { lich über 15 J. }\end{array}$} & \multicolumn{2}{c|}{$\begin{array}{c}\text { Realkapital (Gebäude } \\
\text { und Maschinen) }\end{array}$} & \multicolumn{2}{c|}{$\begin{array}{c}\text { Landw. Kulturfläche } \\
\text { (ohne Alpweide), ha }\end{array}$} \\
\hline & absolut & Index & Mio Fr. real & Index & absolut & Index \\
\hline 1955 & 274863 & 100 & 2000 & 100 & 1254153 & 100 \\
\hline 1960 & 232667 & 88 & 2203 & 110 & & - \\
\hline 1965 & 183102 & 66 & 2182 & 109 & 1213420 & 97 \\
\hline 1969 & 161152 & 58 & 2210 & 110 & 1207837 & 96 \\
\hline 1975 & 131383 & 48 & 1614 & 81 & 1173855 & 94 \\
\hline 1980 & 121372 & 44 & 1845 & 92 & 1202006 & 96 \\
\hline 1985 & 113748 & 41 & 2077 & 104 & 1199811 & 96 \\
\hline 1990 & 97349 & 35 & $2258^{2}$ & 113 & 1202357 & 96 \\
\hline
\end{tabular}

${ }^{1}$ Schätzung aufgrund der sektoralen Einkommensrechnung

21988

Quelle: SBS, Statistische Erhebungen und Schätzungen, div. Jg.

\section{Die Ursachen des Strukturwandels in der Vergangenheit}

Abbildung 1 zeigt, dass seit 1955 die Anzahl der Betriebe um fast $50 \%$ zurückgegangen ist. Die Zahl der männlichen landwirtschaftlichen Arbeitskräfte ist im gleichen Zeitraum gar um 65 \% gesunken. Der Faktor Boden hat insge- 
samt nur etwa $3 \%$ abgenommen, jedoch eine stete Umverteilung zu grösseren Betriebseinheiten erfahren. Rund $45 \%$ der kleinflächigen Betriebe bewirtschaften nur etwa acht Prozent der Fläche. Der Realkapitalbestand ist zeitweise gewachsen, dann wieder gesunken und somit real in etwa gleich gross geblieben. Zusammenfassend könnte man vereinfacht sagen: Weniger Betriebe und Arbeitskräfte bei fast gleichviel Boden und Realkapital. Diese Aussagen sollen nun aber verfeinert werden, indem die einzelnen Ursachen des Strukturwandels analysiert werden.

Am Anfang unserer Argumentationskette stehen die Arbeitskräfte bzw. die Landwirte oder Bauern und Bäuerinnen, aber auch landwirtschaftliche Angestellte. Die Zahl der Bauernbetriebe verändert sich in rund $90 \%$ aller Fälle, indem Söhne oder Töchter nicht mehr Nachfolger ihrer bäuerlichen Väter werden. Diese Abnahmen betrugen in den achtziger Jahren rund $2 \%$, in den sechziger Jahren 4 bis $5 \%$, dazwischen in den Rezessionsjahren etwa $1.3 \%$. Diese Unterschiede sind eine Funktion des Sogs, den die jeweilige ausserlandwirtschaftliche Arbeitsmarktlage auf die potentiellen Nachfolger ausübte. Dadurch entstand auch eine in allen Ländern feststellbare leichte Überalterung des Bauernstandes. Bauern, die 30-jährig und älter sind, geben den Landwirtschaftsberuf in der Regel nur dann auf, wenn sie am gleichen Wohnort eine ihnen zusagende Beschäftigung finden. Dieses Abwanderungsmuster erklärt nun seinerseits die in Abbildung 1 dargestellten Veränderungen der Flächengrössenklassen. Ein Teil der Fläche aufgebender Betriebe dient den Haupterwerbsbetrieben zur Flächenvergrösserung. Aber auch am Wohnort verbleibende landbesitzende nichtbäuerliche Nachkommen bewirtschaften wegen der Direktzahlungen zunehmend ihre eigenen Flächen. Das Gleiche gilt für Rentnerbetriebe, die in der Landwirtschaft eine sinnvolle Beschäftigung finden. So erklärt sich die noch heute relativ grosse Anzahl von rund $45^{\prime} 000$ Kleinstbetrieben unter 5 Hektaren. Diese Anzahl ist im europäischen Vergleich relativ betrachtet sehr hoch, weil die Schweiz eine vergleichsweise sehr dezentralisierte allgemeine Wirtschaftsstruktur aufweist.

Die Folge des beschriebenen Abwanderungsmuster ist, dass die Flächen der Haupterwerbsbetriebe durch Zupacht allmählich steigen, jene der Nebenerwerbsbetriebe jedoch kaum. Nebenerwerbsbetriebe bewirtschaften oft Weinberge, kleine Gemüseanlagen oder Flächen für einige Ziegen oder Schafe und nur in wenigen Fällen grössere Ackerflächen. Somit behindert eine allzu starke agrarpolitische Förderung von Kleinbetrieben notwendige Strukturanpassungen von Haupterwerbsbetrieben.

Mit diesem Wandel geht stets eine Umschichtung des Realkapitals einher. Einerseits wurden in den frühen Nachkriegsjahren landwirtschaftliche Angestellte durch Maschinen ersetzt, womit Haupterwerbsbauern ihre Arbeitsproduktivität steigern konnten. Gleichzeitig führten Betriebsvergrösserungen dazu, dass mit weniger Maschinen grössere Flächen bearbeitet und Tierbestände betreut werden konnten. Somit sind in der Regel kleinere Betriebe pro Flächeneinheit sowohl arbeits- als auch kapitalintensiver als grössere. 
Begriffe, die nicht mehr unter den Strukturbegriff fallen, aber in unserer Zeit oft verwendet werden, sind Produktivität und Intensität. Durch den oben beschriebenen Strukturwandel ist insbesondere die bereinigte Arbeitsproduktivität jährlich um 4 bis $6 \%$ angestiegen. Gleichzeitig erfuhr die Schweiz in der Nachkriegszeit die weltweit grössten flächenbezogenen Intensitätssteigerungen. Unter Intensität versteht man Faktorverhältnisse, also zum Beispiel Tiereinheiten pro Flächeneinheit. Der Grund dafür liegt in den sehr hohen Agrarproduktpreisen im Verhältnis zu den relativ tiefen Preisen von ertragssteigernden Hilfsstoffen wie Dünger, Pflanzenschutz und zugekauften Futtermitteln. Bekanntlich führten diese Intensitätssteigerungen zu ökologischen Gefährdungen und zur Tierschutzproblematik. Mit der neuen Agrarpolitik seit 1992 sind diesbezüglich die Weichen neu gestellt worden.

\section{Schweizer Agrarstrukturen im europäischen Vergleich}

Im europäischen Vergleich weist die Schweiz eine sehr kleine durchschnittliche flächenbezogene Betriebsgrösse auf. Sie betrug 199011.4 Hektaren im Vergleich zu 16.8 in Westdeutschland, 28.6 in Frankreich, 15.3 in Holland (viele Gartenbaubetriebe), 32.2 in Dänemark und 64.4 in Grossbritannien, jedoch nur 12.8 in Österreich. Diese Zahlen zeigen, dass bei den Standardbetrieben in der Schweiz europäisch gesehen nur die grösseren annähernd wettbewerbsfähige Strukturen aufweisen. Dass Betriebe allerdings auch durch Spezialisierungen und Nischenproduktion Grössennachteile überwinden können , sei hier nur am Rande erwähnt.

\section{Strukturwandel in der Zukunft}

Angenommen, dass die Agrarpolitik den nun eingeschlagenen Weg vom Konzept „Agrarpolitik 2002“ weiterverfolgt, was nach der Volksabstimmung vom 9. Juni 1996 höchst wahrscheinlich ist, lässt sich auch der zu erwartende zukünftige Strukturwandel mehr oder weniger voraussagen. Wir kennen hierfür die heutige Altersstruktur der Haupterwerbslandwirte, deren Nachfolgeverhältnisse und die Betriebsgrössen. Ungewissheit besteht über die Entwicklung der wirtschaftlichen und politischen Rahmenbedingungen und somit über die Sogwirkung auf die im Beruf stehenden Landwirte und deren Nachkommen. Im Gutachten über die Auswirkungen des GATT - Vertrages von 1994 auf die Landwirtschaft gingen wir von einer jährlichen Abnahme von $2 \%$ der auf Vollerwerbsbetriebe umgerechneten Einheiten aus. Diese Rate entspricht jener der achtziger Jahre, also einer Zeit mit guter Konjunkturlage. Da die allgemeinen Wirtschaftsaussichten zur Zeit eher ungünstig eingeschätzt werden, ist damit zu rechnen, dass der Sog eher abnimmt. Doch selbst wenn der Sog gegen Null ginge, lässt sich sagen, dass bei bekannter Nachfolgesituation die Haupterwerbsbetriebe pro Jahr etwa um $1.5 \%$ abnehmen werden. Innerhalb von zehn Jahren betrifft dies unter den genannten Annahmen die Aufgabe von rund 9'000 Betrieben, ohne dass von Bauernsterben die Rede sein muss. Dem geringeren Sog steht der oben genannte Umbau der Agrarpolitik gegenüber, der für mittelgrosse, noch intensiv oder zu wenig ökologisch produzierende 
Haupterwerbsbetriebe die Einkommensdifferenzen zur übrigen Wirtschaft vergrössern könnte. Diese Betriebe werden auf Spezialprodukte umstellen oder die Produktion aufgeben. Die Statistik zeigt, dass aber viele dieser mittelgrossen Haupterwerbsbetriebe im Talgebiet ohnehin zur oben erwähnten Kategorie der auslaufenden Betriebe gehören. Andererseits gehören jüngere Haupterwerbsbauern in der Regel zur Kategorie mit bereits grösseren Betriebsflächen, sowohl im Tal- als auch im Berggebiet.

Die Annahme einer etwas nachlassenden Sogwirkung bei gleicher oder schwach reduzierter Agrarstützung (was bei anhaltender Rezession auch eintreten könnte), wirkt sich auch auf die Anzahl und die Strukturen der Nebenerwerbsbetriebe aus. Untersuchungen im Kanton Uri haben gezeigt, dass ein positiver Zusammenhang zwischen der allgemeinen Wirtschaftslage (dort Bauwirtschaft) und der Zahl von Nebenerwerbsbetrieben besteht. Bei gesicherten und relativ hohen ausserlandwirtschaftlichen Löhnen können es sich mehr Leute leisten, nebenbei eine relativ unrentable und arbeitsaufwendige kleine Landwirtschaft zu betreiben. Auf solchen Betrieben wird in der Regel mit Geld investiert, das ausserhalb der Landwirtschaft verdient wird. Daneben wird es auch in Zukunft eine eher zunehmende Anzahl von Rentnerbetrieben geben, obwohl wichtige Direktzahlungen an über 65-jährige Landwirte nicht mehr ausbezahlt werden. Haupt- und Nebenerwerbsbetriebe weisen somit unterschiedliche Ursachen ihrer Existenzen bzw. deren Veränderungen auf. Haupterwerbsbetriebe sind im Zeitablauf wirtschaftlich auf Möglichkeiten von Strukturveränderungen angewiesen, nicht jedoch Nebenerwerbsbetriebe.

\section{Agrarpolitik 2002: Ökonomische und ökologische Auswir- kungen auf die Regionen der Schweiz}

\section{Agrarpolitischer Umbau}

Seit 1992 ist die schweizerische Agrarpolitik in einem markanten Umbau. Dieser umfasst folgende drei Schritte:

- Abänderung des (alten ) Art. 29 des Landwirtschaftsgesetzes und seine Ergänzung durch die (neuen) Artikel 31a und 31b

- Beitritt zum neuen WTO/GATT - Vertrag (Ergebnis der Uruguayrunde)

- Vollständiger Umbau des Landwirtschaftsgesetzes, bekannt unter der Bezeichnung Agrarpolitik 2002 (AP 2002).

Diese Neuorientierung der schweizerischen Agrarpolitik hat eine interne und eine externe Ursache. Die interne Ursache besteht darin, dass wegen der bis 1992 gewährten kostendeckenden Preise, des perfekten Aussenhandelsschutzes und der Exportsubventionen folgende Probleme entstanden:

- Kaum finanzierbare Marktüberschüsse,

- eine umweltgefährdende Produktionsintensität und 
- interventionistisch bedingte Einkommensunterschiede innerhalb der Landwirtschaft (Berg-/Talgebiet, kleinere/grössere Betriebe, Milch/Fleischproduktionsbetriebe)

Die externe Ursache lässt sich mit folgenden Stichworten angeben: Internationale Agrarmarktüberschüsse infolge des Agrarprotektionismus der Industrieländer; totaler Preiszusammenbruch auf den Agrarmärkten in den achtziger Jahren; zunehmende Armut in vielen Entwicklungsländern; Zusammenbruch der Planwirtschaften. Dies waren bekanntlich die Hauptgründe für die Uruguayrunde im Rahmen des GATT.

Nach diesen Bemerkungen treten wir nun auf die Frage ein, ob die oben genannten Gesetzesänderungen spezifisch ökonomische und auch ökologische Auswirkungen auf die Regionen, insbesondere den Alpenraum der Schweiz, haben bzw. noch haben werden.

\section{Ergänzung des Landwirtschaftsgesetzes mit den Artikeln 31a und 31b von 1992}

Mit dieser Gesetzesveränderung wurde die Trennung der Produktpreis- von der Einkommenspolitik für die ganze Landwirtschaft vollzogen. Ab jenem Zeitpunkt wurde der produktbezogene Agrarschutz (Milchpreise, Getreidepreise, etc.) schrittweise gesenkt und die Einkommen zunehmend über ergänzende produktionsunabhängige Direktzahlungen ausgeglichen. Weil diese Direktzahlungen produktionsunabhängig sind, also an Betriebe und an die Flächen gebunden, führte bereits dieser Umbau auch zu regional unterschiedlichen Auswirkungen. Produktionsintensive Betriebe hatten durch den Preisabbau relativ grössere Verluste als extensiv produzierende, während alle die gleichen Zahlungen pro Fläche bzw. pro Betrieb erhielten. Die Absicht dieser Gesetzesänderung war, eine zwar flächendeckende, aber extensivere Landwirtschaft herbeizuführen. Abbildung 2 zeigt, wie sich seither diesbezüglich die Bundesausgaben tatsächlich von der Produktionsstützung auf Direktzahlungen verlagerten. 
Abb. 2: Umbau der Agrarstützung seit 1990

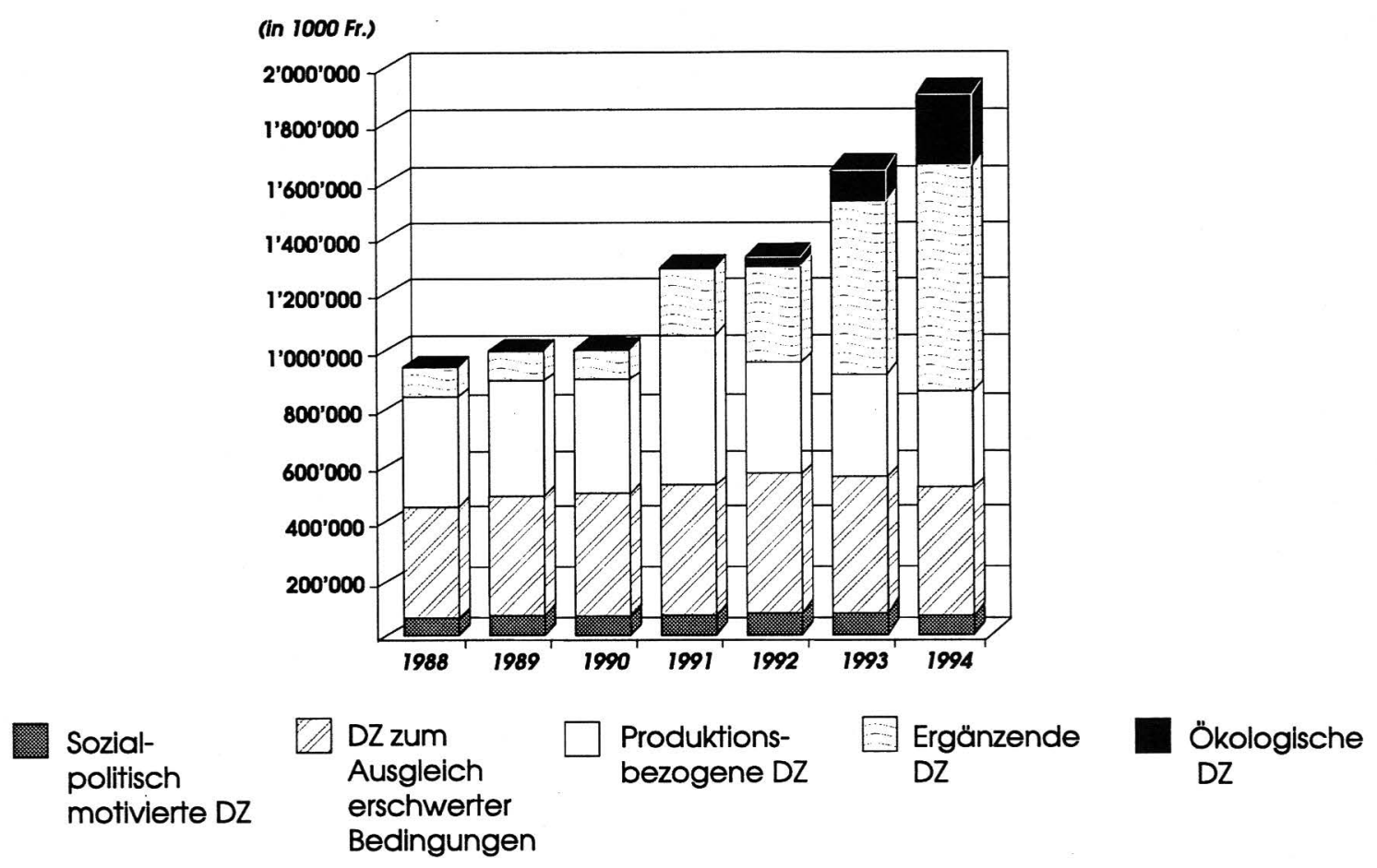

Quelle: Stabsstelle Agrarwirtschaft, BLW

Stand der Zahlen:

26.1.1994

$\mathrm{Ob}$ die Agrarstrukturen auf diesen Umbau auch erwartungsgemäss reagiert haben, lässt sich wegen der wenigen Jahre erst durch gezielte Einzeluntersuchungen aufzeigen. Wir haben solche an den verfügbaren Daten in den Kantonen Bern und Graubünden vorgenommen. Die Ergebnisse enthält Tabelle 2.

Tab.2: Veränderungen der Anzahl Betriebe 1990 bis 1994 in den Kantonen Bern und Graubünden

\begin{tabular}{|l|c|l|c|}
\hline Kanton Bern & $\begin{array}{c}\text { \% Veränderung } \\
1990-1994 \text { jährl. }\end{array}$ & Kanton Graubünden & $\begin{array}{c}\% \text { Veränderung } \\
1990-1994 \text { jährl. }\end{array}$ \\
\hline Talzone & -3.4 & Talzone & -5.8 \\
\hline Voralp. Hügelzone & -2.8 & Voralp. Hügelzone & -7.5 \\
\hline Bergzone 1 & -2.3 & Bergzone 1 & -5.3 \\
\hline Bergzone 2 & -2.3 & Bergzone 2 & -2.8 \\
\hline Bergzone 3 & -2 & Bergzone 3 & -4.5 \\
\hline Bergzone 4 & Bergzone 4 & +0.2 \\
\hline
\end{tabular}

Aus den beiden Teilen in Tabelle 2 lassen sich gleiche Tendenzen herauslesen. Ausgehend von einer erwarteten durchschnittlichen jährlichen Abnahme der Anzahl Betriebe von zwei Prozent stellt man fest, dass die Abnahmen in den intensiv produzierenden Talregionen grösser als in den extensiver 
produzierenden Bergzonen sind. Diese, wenn auch als provisorisch zu betrachtenden Veränderungen, weisen zumindest darauf hin, dass regional unterschiedliche Tendenzen zu erwarten sind; auch dürfte die Agrarproduktion tendenziell ökologischer werden und die Agrarüberschüsse abnehmen. Bekannt ist ferner, dass relativ viele Landwirte auf die speziellen ökologischen Beiträge nach Art. 31b reagiert haben bzw. ihre Betriebe auf diese Produktionsformen umgestellt haben (Baur, 1995).

\section{GATT-bedingte regionale Auswirkungen}

Die wichtigsten drei agrarbezogenen Verpflichtungen der Uruguayrunde des GATT sind in Tabelle 3 zusammengestellt. Erstens müssen die Agrarpreise gesenkt werden, zweitens muss ein minimaler Marktzutritt für Agrarprodukte gewährt werden und drittens sind die Exportsubventionen zu kürzen. Besonders zu beachten ist, dass der Agrarschutz nur dort abgebaut werden muss, wo keine Produktionsbeschränkungen angewendet werden (Kontingentierungen). Ferner sind (in der gleichen Kolonne aufgeführt) produktunabhängige Zahlungen nach wie vor möglich (Green Box).

\section{Tab. 3: Elemente der Schlussakte der Uruguay-Runde im Agrarbereich}

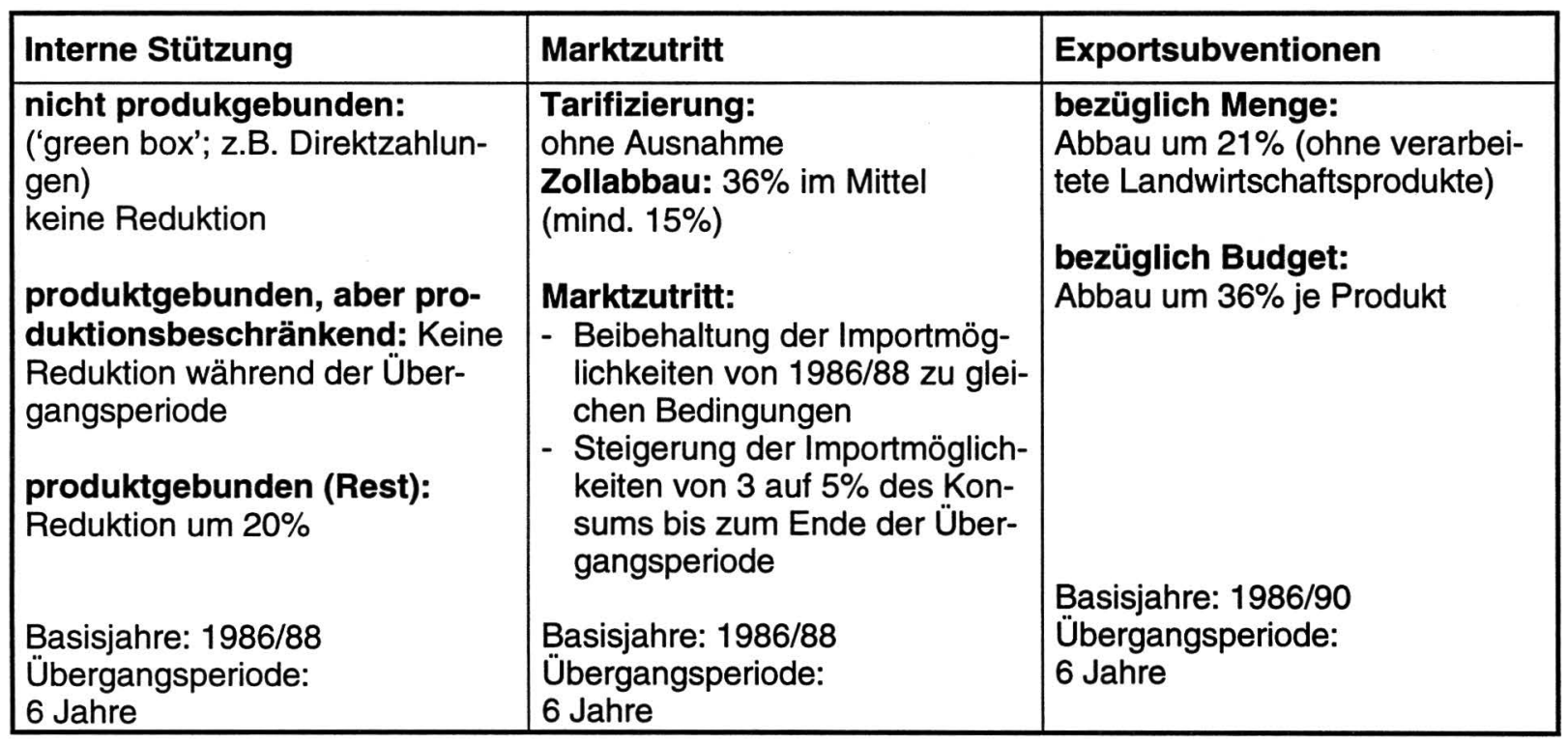

Quelle: Bundesamt für Landwirtschaft

Zu den Auswirkungen der GATT-Beschlüsse wurden sehr ausführliche Untersuchungen angestellt (Rieder, et al. 1995). Folgende Ergebnisse sind für die hier vorliegende Fragestellung relevant:

- die weitere Senkung der Agrarpreise und deren teilweiser Ausgleich durch Direktzahlungen wird die bereits oben erwähnte Tendenz der relativen Bevorzugung extensiv produzierender Betriebe im Vergleich zu eher intensi- 
ven Betrieben verstärken. Konkret bedeutet dies eine relative Bevorzugung des Berggebietes und der Regionen mit eher grösseren Betrieben. (Westschweiz).

- weil im Laufe der Anpassung bis zum Jahre 2002 die Produktpreise gesenkt und die Direktzahlungen ausgebaut werden sollen, interessiert die Frage der dannzumaligen Zusammensetzung des Gesamterlöses der Landwirte in verschiedenen Regionen. Nach unseren Berechnungen (Rieder et al. 1995) schwanken diese Anteile von rund $8 \%$ im Talgebiet bis zu rund $20 \%$ Prozent in den Bergzonen 2-4. Hier ist erklärend beizufügen, dass diese Direktzahlungen aus verschiedenen Einzelabgeltungen für umweltrelevante Leistungen bestehen. Jede hat ihr Ziel. Die Landwirte sind sich dessen bewusst und reagieren auch gezielt auf die einzelnen Direktzahlungsarten. So ist auch der relativ hohe Anteil in den Bergzonen 2-4 ökonomisch unproblematisch und argumentativ folgendermassen zu rechtfertigen:

- durch die GATT-bedingten geringeren Exportbeiträge für Zucht- und Nutzvieh sind vor allem die traditionellen Viehexportregionen im Berggebiet betroffen. Die Preise dürften tatsächlich sinken, jedoch soll ein gleichwertiger Ausgleich über Direktzahlungen erfolgen.

- von den Kürzungen der Exportbeiträge für Käse sind vor allem die intensiven Milchproduktionsbetriebe im Voralpengebiet betroffen. Aber auch hier ist vorgesehen, durch Direktzahlungen die bereits eingeleiteten Milchpreissenkungen zu einem grossen Teil auszugleichen.

\section{Agrarpolitik 2002}

Und schliesslich wird zur Zeit das ganze Landwirtschaftsgesetz neu geschrieben.

Diese neue „Agrarpolitik 2002“ sieht nun vor, dass im Rahmen des im GATT vereinbarten Aussenhandelsschutzes im Inland Marktpreise herrschen sollen. Das Gesetz spricht von Richtpreisen, die angestrebt werden. Allerdings sind "Rettungsmassnahmen" vorgesehen, mittels derer gesorgt wird, dass diese Richtpreise mehr oder weniger erreicht werden. Um verbleibende Überschüsse abzusetzen, sind bei der Milch Zulagen auf verkäster Milch vorgesehen, bei Raps und Zucker Beiträge an die Fabriken für deren gemeinwirtschaftlichen Leistungen. Der Staat wird aber keine direkten Markteingriffe mehr wie bisher vornehmen. Daher werden Preise je nach interner Marktlage gelegentlich auch stark fallen bzw. steigen. Die Preise sollen also Angebot und Nachfrage steuern. Nun stellt sich die Frage, ob aus der Sicht der relativ heterogenen Agrarregionen der Schweiz damit Regionalprobleme verbunden sind, und ob man gar an regionalpolitische Massnahmen zu denken hätte.

Mit der neuen Agrarpolitik fallen fast alle staatlichen und halbstaatlichen Eingriffe auf den Märkten weg. An dessen Stelle tritt Marktfreiheit. Mit anderen Worten, jeder Landwirt soll das machen, was er für sich am günstigsten hält. 
Zwei Elemente werden dadurch neu sehr wichtig, nämlich die Lage seines betrieblichen Standorts einerseits und das Absatzpotential für seine Produkte. Beide Elemente sind für jeden einzelnen Landwirt grundsätzlich verschieden. Somit lässt sich auch nur noch theoretisch weiter argumentieren, was mögliche Auswirkungen auf einzelne Regionen sein werden. In gewissen Regionen wird die Belieferung von regionalen Märkten das beste sein; an anderen Orten ist die Produktsammlung durch regionale Genossenschaften, die die Güter auf grössere Märkte bringen, das Angebrachte. Touristenregionen mögen saisonale Absatzmärkte haben; andere Regionen haben andere spezifische Marktund Produktionsvorteile.

\section{Zur Nachhaltigkeit der Landwirtschaft unter regionalen Aspekten}

Die neue Agrarpolitik 2002 soll optimale Rahmenbedingungen festlegen, durch die eine private Landwirtschaft auch die öffentlichen Ziele der Gesellschaft erfüllt. Dazu sind die oben beschriebenen Massnahmen nötig. Diese Massnahmen haben effizient, effektiv und auch sozialverträglich zu sein. Darauf sei hier nicht weiter eingetreten, bzw. für die folgenden Ausführungen interessiert uns hier vielmehr die Frage, ob man die beschriebenen neuen agrarpolitischen Konzepte auch mit den regionalen Erfordernissen des Nachhaltigkeitskonzepts (nach RIO92) in Übereinstimmung bringen kann. Was muss sich diesbezüglich gegenüber der Vergangenheit konkret ändern? Kann eine Berglandwirtschaft überhaupt am Nachhaltigkeitskonzept gemessen werden?

\section{Zum Konzept der Nachhaltigkeit}

Das Konzept der Nachhaltigkeit enthält bekanntlich eine

- ökologische

- ökonomische (volkswirtschaftliche) und

- soziale Dimension.

Das Konzept verlangt, dass gleichzeitig alle drei Dimensionen minimale Standards aufzuweisen haben, oder dass Verbesserungen der einen Dimension nicht auf Kosten einer anderen erfolgen (sogen. Win-Win Konzept der Weltbank; Serageldin 1995). Die alte Agrarpolitik hat ganz eindeutig sowohl die ökologische wie auch die volkswirtschaftliche Komponente vernachlässigt und ihr Schwergewicht auf eine sozial motivierte Erhaltungspolitik gelegt. Diese Politik hat zweifellos zu regionalen Strukturen geführt, die im positiven Sinne ländliche Kulturlandschaften prägten. Das Konzept war aber nicht nachhaltig, was die oben erwähnten Probleme belegen. Somit stellt sich zukunftsorientiert die Frage, ob die neue Agrarpolitik die volkswirtschaftliche und die ökologische Dimension verbessert, ohne die soziale zu verschlechtern. Darauf wollen wir zuerst eine allgemeine und dann eine regionenbezogene Antwort geben. 


\section{Zur Nachhaltigkeit der neuen Agrarpolitik}

Im allgemeinen kann gesagt werden, dass durch den Umbau der Agrarpolitik (inkl. des Gewässerschutzgesetzes) bei allen drei Dimensionen Verbesserungen oder zumindest keine Verschlechterungen entstehen. Die ökologischen Verhältnisse werden verbessert, indem durch Produktpreissenkungen die ökonomischen Anreize reduziert werden, zu intensiv zu produzieren. Das Gewässerschutzgesetz und die spezifischen Beiträge nach Artikel 31b des Landwirtschaftsgesetzes wirken in die gleiche Richtung. Ökonomisch bzw. volkswirtschaftlich wird sich die Situation verbessern, weil nach und nach die Marktkräfte zu effizienteren Produktionsstrukturen führen werden. Die soziale Dimension wird auf gezielte Weise über die vorgesehenen Direktzahlungen angestrebt, indem diese wie ein soziales Auffangnetz wirken, ohne den Markt zu stören.

Die regionsbezogene Antwort zur Nachhaltigkeit der anzustrebenden Agrarstrukturen geben wir, indem wir auf die Aktionsparameter zurückgreifen, die der Staat auch unter der neuen Agrarpolitik in seiner Kompetenz behält. Dazu soll Abbildung 3 behilflich sein. Diese bringt zu Ausdruck, dass der Strukturwandel von vier Determinanten abhängt, nämlich den Produktpreisen, den regionalen Arbeitsmarktverhältnissen, den Direktzahlungen und den Struktur Anpassungsmassnahmen. Die Produktpreise sind, wie oben dargelegt, nicht mehr im Kompetenzbereich des Staates. Die regionalen Arbeitsmarktbedingungen, die von Region zu Region sehr unterschiedlich sein können, liegen auch nicht im Einflussbereich der Agrarpolitik, allenfalls der regionalen Wirtschaftspolitik. Dem Staat verbleiben die zwei Aktionsbereiche Direktzahlungen und Strukturmassnahmen. Mit diesen beiden Massnahmen hat der Staat die Möglichkeit, regional unterschiedlich zu wirken. Er kann diese nach ungleichen Kriterien anwenden und so auf Besonderheiten unterschiedlicher Agrarstrukturen in einzelnen Regionen Rücksicht nehmen. Durch geschickte Kombination dieser zwei Massnahmengruppen kann er wesentlich beitragen, dass sich die Forderungen nach Nachhaltigkeit effizienter und auch effektiver als bisher erreichen lassen. So wird es grundsätzlich möglich, gleichzeitig sowohl ökologische wie auch ökonomische Forderungen zu erfüllen. Im Sinne eines Leitbildes sprechen wir dann von einer Ökologischen Wettbewerbslandwirtschaft. 
Abb. 3: Determinanten des Strukturwandels

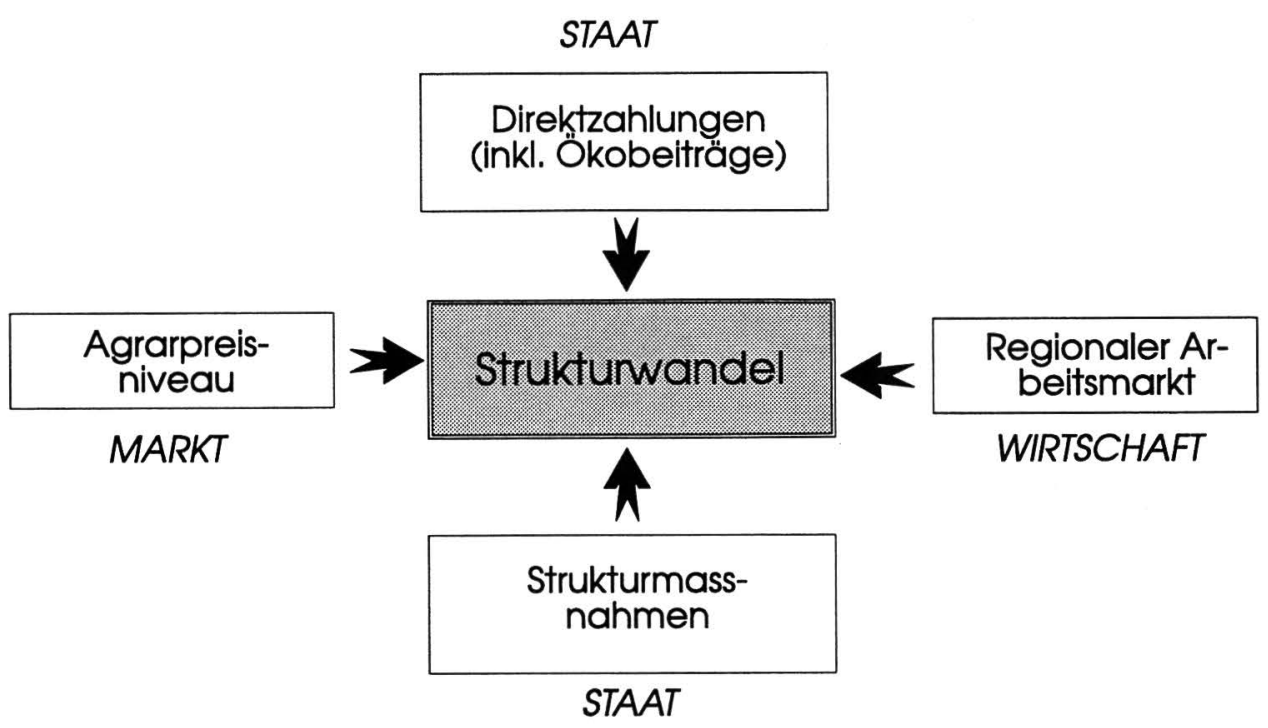

\section{4. Ökologische Wettbewerbslandwirtschaft: ein landwirt- schaftliches Leitbild auch für den Alpenraum?}

Am Ende des vorangehenden Abschnittes haben wir den Begriff Ökologische Wettbewerbslandwirtschaft als Leitbild eingeführt. Dieser Begriff wurde vom Autor und Mitarbeiterinnen (Baur et al.1995)) entwickelt, als man für die Landwirtschaft des Kantons Zürich eine Synthese von Ökologie und Ökonomie suchte. Das Leitbild der Ökologischen Wettbewerbslandwirtschaft wurde dort drei Alternativen gegenübergestellt, wie dies aus der Tabelle 4 hervor geht. Das Leitmotiv, alternative Leitbilder zu entwickeln, war, ein Leitbild zu entwickeln, bei dem sowohl die ökonomischen als auch die ökologischen Anforderungen an die Landwirtschaft in gesellschaftlich gefordertem Ausmass erfüllt werden.

Tab. 4: Vier mögliche Leitbilder zur Landwirtschaft der Schweiz

\begin{tabular}{|l|l|l|l|l|}
\hline & $\begin{array}{c}\text { Weg I } \\
\text { Produktionsland- } \\
\text { wirtschaft }\end{array}$ & $\begin{array}{c}\text { Weg II } \\
\text { Ökologische } \\
\text { Produktions- } \\
\text { landwirtschaft }\end{array}$ & $\begin{array}{c}\text { Weg III } \\
\text { Ökologische } \\
\text { Wettbewerbs- } \\
\text { landwirtschaft }\end{array}$ & $\begin{array}{c}\text { Weg VI } \\
\text { Bereitstellungs- } \\
\text { landwirtschaft }\end{array}$ \\
\hline Oberziel & $\begin{array}{l}\text { Erhaltung mög- } \\
\text { lichst vieler Betrie- } \\
\text { be und der bäuer- } \\
\text { lichen Kultur }\end{array}$ & $\begin{array}{l}\text { Erhaltung mög- } \\
\text { lichst vieler Betrie- } \\
\text { be und der bäuer- } \\
\text { lichen Kultur sowie } \\
\text { Förderung einer } \\
\text { umweltverträgli- } \\
\text { cheren } \\
\text { Landwirtschaft }\end{array}$ & $\begin{array}{l}\text { Erhaltung einer } \\
\text { flächendeckenden, } \\
\text { wettbewerbs- } \\
\text { fähigen und um- } \\
\text { weltverträglicheren } \\
\text { Landwirtschaft }\end{array}$ & $\begin{array}{l}\text { Erhaltung einer } \\
\text { minimalen Produk- } \\
\text { tionsbereitschaft }\end{array}$ \\
\hline
\end{tabular}


Beim Leitbild Produktionslandwirtschaft (Weg I) handelt es sich um den Weg, den die Agrarpolitik der Schweiz seit 1950 rund dreissig Jahre lang betrieben hat. Erhaltung möglichst vieler Betriebe stand im Vordergrund. Die Strategie bestand in der Einkommenssicherung über Preise und in bescheidenem Ausmass in Kostensenkungsmassnahmen. Dieser Weg hat zu den in diesem Artikel weiter oben beschriebenen Problemen ökologischer und ökonomischer Art geführt.

Die zweite Alternative eines Leitbildes heisst Ökologische Produktionslandwirtschaft. Im Vergleich zu Weg I bleibt hier die Erhaltung möglichst vieler Betriebe und einer herkömmlichen bäuerlichen Kultur, wird aber ergänzt durch eine spezifische Förderung eine umweltverträglichen Landwirtschaft. Der Kostenseite wird dabei kaum Beachtung geschenkt, während andererseits so hohe Ökologisierungsbeiträge gefordert werden, bis die Betriebe auch ihre Einkommensansprüche befriedigt haben. Einkommenspolitik wird also unter dem Deckmantel der Ökologisierung betrieben. Die entsprechende Politik ist in der Schweiz in etwa von Mitte der achtziger Jahre bis heute betrieben worden. Artikel $31 \mathrm{~b}$ des Landwirtschaftsgesetzes und die erste abgelehnte und die zweite noch hängige Kleinbauerninitiative entsprechen grosso modo diesem Leitbild.

Eine weitere Alternative besteht in einer Bereitstellungslandwirtschaft als Leitbild. Auf diesem Weg würde man nur noch eine Produktionsbereitschaft sicherstellen. Strukturerhaltung und Einkommenssicherung als Teilziele liesse man fallen. Entsprechend würde sich die Agrarpolitik auf weit geringere Eingriffe begrenzen. Es würden von selbst marktgerechte Agrarstrukturen entstehen. Dieser Weg wird zum Vergleich immer wieder in die Diskussion eingebracht, sie liegt aber bisher ausserhalb aller politischen Willensäusserungen des Schweizervolkes zur Agrarfrage.

In den drei skizzierten Alternativen von Leitbildern ist nirgends die Forderung, dass sowohl ökologische als auch ökonomische Ziele gleichzeitig erfüllt sein müssen, aufgestellt worden. Dies tun wir jedoch mit dem Leitbild der Ökologischen Wettbewerbslandwirtschaft. Als Ziel verlangt dieser Weg die Erhaltung (oder Wiederherstellung) einer flächendeckenden, wettbewerbsfähigen und gleichzeitig umweltverträglichen Landwirtschaft. Auf der Massnahmenebene verlangt dieser Weg, den Strukturwandel stärker zu fördern und verschiedene Massnahmen zu reduzieren oder abzuschaffen, andererseits aber neue Massnahmen, die zu einer gezielten Ökologisierung der gesamten Landwirtschaft führen, einzuführen.

Der Forderung, auch im Berggebiet die Landwirtschaft nach dem Leitbild der Ökologischen Wettbewerbslandwirtschaft auszurichten, kann entgegengehalten werden, es vernachlässige die natürlichen und topographischen Nachteile des Alpenraumes, oder aber es reduziere die Anzahl der Landwirte zu stark, und es wäre schon gar nicht geeignet für die vielen Nebenerwerbsbetriebe, die es im Berggebiet gebe. Diesen berechtigten Einwendungen können folgende 
Argumente entgegengestellt werden: Den ökologischen Anforderungen an die Landwirtschaft im Alpenraum kommt unseres Erachtens eine sehr grosse Bedeutung zu. Erstens sind dort viele Böden fragiler als im Flachland; um so wichtiger ist es, dass in jenen Gebieten weder eine Übernutzung noch eine Unternutzung satt findet. Weder Hangstabilitäten, die Biodiversität noch kleine oder grössere Gewässer dürfen gefährdet werden. Neben diesen physischen Aspekten der Alpenlandwirtschaft gibt es ihren ästhetischen Wert. Dieser schlägt sich in den Landschaftsbildern nieder, die zum schweizerischen Kulturgut gehören und beim nichtbäuerlichen Bewohner unseres Landes einen eindeutigen Optionswert besitzen. Gerade weil dem so ist, sind vorhandene heutige Übernutzungen und teilweise auch Unternutzungen mit geeigneten Massnahmen zu korrigieren. Soviel zum ökologischen Aspekt des Leitbildes. Kann nun der ökonomische Aspekt mit gleicher Verbindlichkeit gefordert werden?

Ökonomisch oder wettbewerbsfähig ist eine Tätigkeit, wenn dabei die Arbeitsproduktivität so gross ist, dass aus dem monetären und naturalen Erlös der Landwirt einen befriedigendes Einkommen erzielt und er dabei normalen Arbeitsbedingungen ausgesetzt ist, eine soziale Anerkennung findet und letztlich in seiner Arbeit eine Sinnerfüllung sieht. Wegen der Schwierigkeiten, diese Ziele aus dem Produkteerlös zu erreichen, gibt es eine Anzahl Direktzahlungen, die den Bergbauern als Entgelt der Produktion öffentlicher Güter ausgerichtet werden. Unter Einbezug beider Einkommenskomponenten soll nach der Leitbildforderung der Landwirt nun ein Einkommen erzielen, das vergleichbar mit den Einkommen seiner dörflichen Mitbewohner sein soll. Diese Forderung ihrerseits ist wieder nur erfüllbar, wenn der Betrieb eine Grösse aufweist, bei der die eingesetzten Maschinen und Gebäude eine gute Auslastung aufweisen und bei der sich dadurch eine befriedigende Arbeitsproduktivität der mitarbeitenden Personen ergibt. Aus den Buchhaltungserhebungen der FAT ist bekannt, dass bei Standardbetrieben im Berggebiet diese Grösse auch dort bei rund 20 Hektaren bzw. Grossvieheinheiten liegt. Sind diese Anforderungen wesentlich unterschritten, so sind Gebäude, Maschinen und Einrichtungen zu wenig ausgelastet, und entweder verdient der Landwirt wirklich zu wenig oder aber er kompensiert diese strukturellen Schwächen durch einen zu grossen Arbeitseinsatz, der nicht mehr normalen Arbeitsbedingungen unserer Zeit entspricht. Gleichzeitig bleibt keine Zeit mehr für soziale Anlässe, und die Sinnerfüllung an der Arbeit geht ebenfalls verloren. In solchen Situationen werden Söhne und Töchter mit grosser Wahrscheinlichkeit nicht Nachfolger auf dem elterlichen Betrieb. Daher ist es so wichtig, dass die technischen Einrichtungen, die Maschinen und Gebäude auch auf den Bergbetrieben immer wieder erneuert werden. Dazu kann der Staat, wie weiter oben dargelegt, finanziell mithelfen. Zudem sind gut strukturierte Betriebe weniger krisenanfällig und überleben gelegentliche Markteinbrüche ohne finanzielle Notlage.

Es bleibt noch die Frage zu beantworten, ob nicht Nebenerwerbsbetriebe dem Leitbild besser entsprechen würden. Hierzu ist vorerst klar zwischen Zuerwerbs- und Nebenerwerbsbetrieben zu unterscheiden. Bei den Zuerwerbsbetrieben handelt es sich um landwirtschaftliche Haupterwerbsbetriebe, die exi- 
stentiell von der Landwirtschaft leben und nur einen relativ kleinen Einkommensanteil ausserlandwirtschaftlich verdienen, zum Beispiel bei Tourismusarbeit im Winter. Für diese Betriebe gilt das vorher Gesagte. Die Nebenerwerbsbetriebe andererseits leben zu einen existentiellen Anteil von nicht-landwirtschaftlicher Tätigkeit, statistisch zu über $50 \%$. Beispiele dafür sind etwa Fabrikarbeiter im Wallis, Wildhüter, Wegmacher und Ähnliche. Bei solchen Betrieben sind erstens in der Regel die Opportunitätskosten der landwirtschaftlichen Arbeitszeit gering oder null, da für diese Person nicht jede Nebenbeschäftigung möglich ist, vielleicht eben nur eine landwirtschaftliche. Daher ist der Verdienst aus der landwirtschaftlichen Tätigkeit auch nicht ausschlaggebend für die nicht-landwirtschaftliche Tätigkeit. Zweitens sind solche Betriebe oft ungenügend mechanisiert bzw. eingerichtet und weisen deshalb keine normale Arbeitsbelastung auf. Somit ist die Nebenerwerbslandwirtschaft direkt abhängig von der örtlichen oder regionalen übrigen Wirtschaft. Wenn die dortigen Beschäftigungsmöglichkeiten stabil sind, kann die Nebenerwerbslandwirtschaft in vielen Fällen über ein Berufsleben einer Person andauern. Selten jedoch weisen solche eigentliche Nachfolger auf. Somit muss die Nebenerwerbslandwirtschaft im Alpenraum bezüglich dem Leitbild der Ökologischen Wettbewerbslandwirtschaft zumindest sehr vorsichtig wenn nicht gar negativ beurteilt werden. Beizufügen bleibt auch hier, dass Nebenerwerbsbetriebe den Strukturanpassungen von Haupterwerbsbetrieben im Wege stehen können.

Zusammenfassend lässt sich daher sagen, dass das Leitbild der Ökologischen Wettbewerbslandwirtschaft mittel- bis langfristig auch im Alpenraum seine volle Berechtigung hat. Im Einzelnen mögen viele Fälle von unseren allgemeinen Standardbetrachtungen zwar abweichen, ab die Kriterien der ökologischen und ökonomischen Anforderungen werden auch diese erfüllen müssen, um langfristig zu überleben.

\section{Politökonomische Probleme bei der Umsetzung von an sich nachhaltigen Lösungsvorschlägen}

In diesem letzten Teil muss die Frage aufgeworfen werden, auf welche möglichen Hindernisse die Umsetzung der oben dargestellten Konzepte stossen könnten. Im zweiten Kapitel haben wir festgehalten, dass eine strukturverändernde Grundströmung durch die Agrarpolitik nicht zu beeinflussen ist. Im Kapitel drei haben wir gezeigt, dass die neue Agrarpolitik durch die verschiedenen Direktzahlungen mittelfristig grundsätzlich den Nachhaltigkeitskriterien gerecht wird. Im vierten Kapitel zeigten wir, dass das Leitbild der Ökologischen Wettbewerbslandwirtschaft an sich für die Landwirtschaft des Alpenraumes auch Gültigkeit hat. Wir haben aber möglicherweise politische Verteilungskonflikte übergangen, weshalb wir darauf in diesem Kapitel explizit eintreten.

Um mögliche politische Spannungsfelder aufzuzeigen, greifen wir zurück auf die drei Komponenten der Nachhaltigkeit und stellen diese im nachfolgenden Schema in Form eines Dreieck dar. Während wir oben fragten, ob der Errei- 
chungsgrad der Ziele verbessert werden konnte, fragen wir hier, welche mögliche Konfliktfelder zwischen den einzelnen Zielen sein könnten, die einer nachhaltigen Landwirtschaft im Alpenraum im Wege stehen könnten. Als „Friede mit der Natur" bezeichnen wir die Beziehung zwischen den ökonomischen und ökologischen Zielen (Jörin, 1996). Vor dem Hintergrund der vielen Umweltprobleme unserer Zeit - auch im Alpenraum - scheinen diese Konflikte

\section{Abb. 4: Nachhaltigkeitsziele im politischen Spannungsfeld}

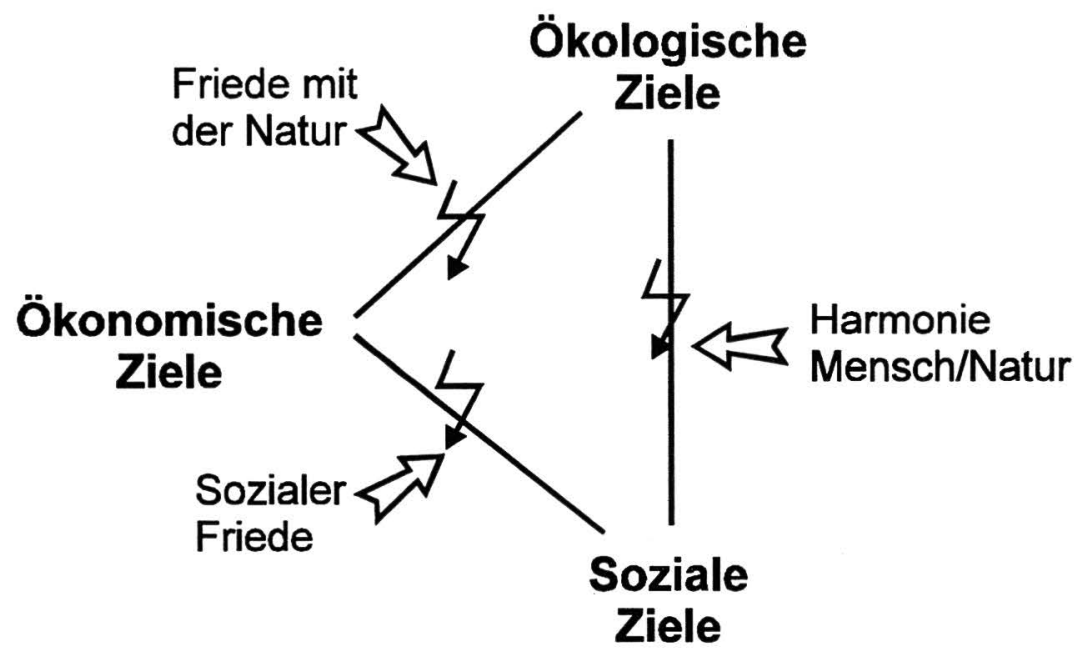

zwischen Ökonomie und Ökologie unüberwindbar. Andererseits haben Umwelt - Ökonomen gezeigt, dass ihre Theorien und Prinzipien auch auf Umweltgüter als knappe Ressourcen anwendbar sind. Man müsse also nur die richtigen Massnahmen im richtigen Ausmass ergreifen, um eine wohlfahrtstheoretisch optimale Menge an Umweltgütern zu erreichen. Dieser Weg ist durch die oben beschriebene Agrarpolitik 2002 grundsätzlich in die Wege geleitet worden. So sind etwa mit den Flächenbeiträgen zur Verhinderung von zu viel Brachland gute Erfolge zu verzeichnen (Anwander et al.). Probleme treten aber dann auf, wenn die Massnahmen auf dieser Beziehung externe Effekte auf andere Bereiche, also auf die Beziehung zwischen ökonomischen und sozialen Zielen, die zweite Achse in unserem Schema, haben. Diese Achse, als „Sozialer Friede" bezeichnet, ist der alte Grundkonflikt zwischen Allokations- und Distributionswirkung einer Massnahme. Wenn also die Ökologische Wettbewerbslandwirtschaft wegen Liberalisierungen auf den Agrarmärkten einen starken Strukturwandel verlangt, um wettbewerbsfähig zu werden, kann diese Tempo Menschen und soziale Dorfstrukturen überfordern. Es kann zu Unterbeschäftigungen kommen. Es kann somit gute Gründe geben, den Anpassungsprozess abzufedern bzw. diesen sozialverträglich zu machen. Bezogen auf unsere Aussagen im dritten Kapitel ist zu sagen, dass es einen optimalen Mix zwischen Strukturanpassungshilfen und sozialer Abfederung durch Direktzahlungen geben muss. Es wäre jedoch nicht konzeptgerecht, mit (zu) hohen Preisstützungen den Produzenten falsche Allokationsanreize zu geben, die sie in wenigen Jahren erst recht in Schwierigkeiten brächten. Die Direktzahlungen 
ihrerseits dürfen aber auch nur so hoch sein, dass sie den Strukturanpassungsprozess nicht bremsen, sondern wie erwähnt nur Verteilungswirkungen haben.

Die Beziehung zwischen sozialen und ökologischen Zielen nennt Jörin (1996) „Harmonie zwischen Mensch und Natur". Im Gegensatz zur Ökonomie, wo es um die Anreize geht, die von aussen her das Verhalten von Menschen beeinflussen (extrinsische Motive), spielen in der Beziehung Mensch/Natur innere Faktoren eine Rolle. Solche Einstellungen der Menschen zur Natur werden mit sogenannten intrinsischen Motiven erklärt. Sozialpsychologisch spricht man hier von Eigenmotivation, von autochtonen Handlungen, dies im Gegensatz zu allochtonem oder „fremdbestimmtem" Verhalten. Überträgt man diese Konzepte auf den Alpenraum, so ist es evident, dass namentlich in der Schweiz sehr vielfältige intrinsische und extrinsische Verhalten nebeneinander existieren. Insbesondere dürtten viele Bürger im und ausserhalb des Alpenraumes der reichen Kultur der Alpenvölker grossen Wert beimessen. Nun ist natürlich diese Kultur an bestimmte Wirtschaftsstrukturen und Traditionen gleichermassen gebunden, wie etwa die Haltung von Ehringerkühen im Wallis (Preiswek, 1986). Insofern befinden wir uns hier an einer interessanten Schnittstelle zu Agrarsoziologie, indem sich die Frage stellt, wie der Strukturwandel das kulturelle Leben in Bergregionen beeinflusst. Die Geschichtsforschung könnte uns diesbezüglich aufzeigen, wie die alpine Gesellschaft in früheren Zeiten mit solchen strukturellen Veränderungen umgingen. Eine grosse Rolle dabei dürfte auch die Tatsache spielen, dass heute die Familiengrössen sich jenen in den Städten angepasst haben und in der Folge viele Dörfer diesbezüglich kritische Einwohnerzahlen bereits unterschritten haben. Daraus wiederum lässt sich folgern, dass gewisse grössere Dörfer noch das Bevölkerungspotential besitzen, ihre Werte in die lokale Gestaltung einer von innen geforderten Nachhaltigkeit einfliessen zu lassen, während viele kleinere Gemeinden bereits zu klein sind, um gestalterisch tätig zu werden und somit von den Wertvorstellungen der Behörden abhängig sind (Häfliger und Rieder, 1996). Welche Nachhaltigkeit bei diesen die geforderte und auch umgesetzte ist, wäre noch zu untersuchen, da die Beamten bzw. die Politiker diese ja mit Steuergeldern abgelten. Hier besteht ein interdisziplinäres Forschungsgebiet, das weitere Grundlagen zu einer nachhaltigen Berggebietspolitik liefern könnte.

\section{Zusammenfassung}

Dieser Artikel setzt sich mit mittel- bis langfristigen Aspekten der Landwirtschaft im Alpenraum auseinander. Der erste Teil analysiert die Grundlagen des landwirtschaftlichen Strukturwandels. Die Haupterkenntnis daraus ist, dass die wichtigsten Ursachen des Wandels ausserhalb des Einflussbereiches der Agrarpolitik liegen, nämlich in der dörflichen und regionalen Entwicklung der übrigen Wirtschaft. Im zweiten Teil wird gefragt, ob die neusten Veränderungen der schweizerischen Agrarpolitik besondere regionale Auswirkungen 
haben. Zur Sprache kommen die neuen Artikel 31a und b des Landwirtschaftsgesetzes, die GATT-Vereinbarungen und der Vorschlag zu „Agrarpolitik 2002“. Wir kommen zum Ergebnis, dass zum Teil bisherige negative Auswirkungen abgebaut werden, zum Beispiel zu hohe Intensitäten und Marktüberschüsse. Gleichzeitig tritt regional betrachtet eine relative Besserstellung der benachteiligten Regionen ein. Dann wird gezeigt, dass die agrarpolitischen Veränderungen auch den Ansprüchen von regionalen Nachhaltigkeiten bezüglich ökonomischen, ökologischen und sozialen Ansprüchen zu genügen vermögen. Strukturverbesserungsmassnahmen und gezielte Direktzahlungen werden als die zwei möglichen staatlichen Handlungsfelder gegeneinander abgewogen. In optimaler Kombination eingesetzt können sie den erforderlichen Strukturwandel auch sozialverträglich machen. In dritten Teil konfrontieren wir die Entwicklungen mit dem Leitbild der Ökologischen Wettbewerbslandwirtschaft. Wir kommen zum Schluss, das dieses Konzept seine Gültigkeit auch für die Landwirtschaft des Alpenraumes hat. Abschliessend fragen wir nach möglichen Schwierigkeiten bei der Umsetzung der geforderten Politik. Politökonomische Hindernisse wie Verteilungsaspekte, Eigeninteressen von Betroffenen und Ähnliches können allenfalls notwendige Änderungen blockieren. Eine saubere Analyse auch dieser Aspekte, unter Einbezug der neuen politischen Ökonomie, führt zu mehr Transparenz und damit auch zur Überwindung kurzsichtiger Handlungsweisen im Interesse langfristiger Lösungen für die Landwirtschaft im Alpenraum.

\section{Literatur}

Anwander, S., S. Bürgi, G. Cavegn, L. Meyer, P. Rieder, J. Salmini (1990): Direktzahlungen an die Berglandwirtschaft. Eine Gesetzesevaluation. vdf, Zürich

Baur, P. (1995): Ökologische Direktzahlungen. Ein Diskussionsbeitrag aus ökonomischer Sicht, in: Agrarwirtschaft und Agrarsoziologie, Schweiz. Gesellschaft für Agrarwirtschaft und Agrarsoziologie, Nr. 2.

Baur, P., S. Anwander und P. Rieder (1995): Ökonomie und Ökologie in der Zürcher Landwirtschaft, vdf.

Häfliger, H., P. Rieder (1996): Optimale Regelungsebene: Fallbeispiel Kulturlandschaft. Synthese 27, Nationales Forschungsprogramm 28, Einsiedeln

Jörin, R. (1996): Strukturwirkungen von Direktzahlungen (Institutsinternes Arbeitspapier)

Preiswerk, Y., B. Crettaz (1986): Das Land, wo die Kühe Königinnen sind, Rotten-Verlags AG, Visp

Rieder, P., Rösti, A., Jörin, R. (1994): Auswirkungen der GATT-UruguayRunde auf die schweizerische Landwirtschaft, Inst. für Agrarwirtschaft, ETH Zürich

Serageldin, I. (1995): Sustainability and the Wealth of Nations: First Steps in an Ongoing Journey, Weltbank, Washington 


\section{$\underline{\text { Schriftenreihe/Publications }}$}

Jahr/ Titel und Autor

Seiten/ Preis/

année Titre et auteur

pages prix

1991/1 Prioritäten und Transparenz in der landwirtschaftlichen 19

Forschung

Peter Rieder

1991/2 Die Erhaltung der bäuerlichen Kulturlandschaft der Alpen agrar- und gesellschaftspolitische Instrumente und Hindernisse Peter Rieder

1991/3 Avenir du monde rural et agriculture

Erwin Stucki

1991/4 Changement de cap en politique agricole, le rôle des

Bernard Lehmann

1991/5 La transformation des systèmes de production dans les Alpes

à la lumière de l'autonomie locale : analyse comparée du Val

Calanca (GR) et du Val de Bagnes (VS)

Erwin Stucki

1991/6 Etude sur le développement équilibré du monde rural en Europe

Erwin Stucki

1992/1 Agrarstrukturentwicklungen in der Schweiz unter Aspekten nationaler Agrarpolitik und laufender internationaler Verhandlungen

Peter Rieder

1992/2 Le développement dans la Vallée des Ormonts: quel rôle pour

l'agriculture de montagne dans le développement local?

Erwin Stucki

1992/3 IER-CERME / Rapport d'activité 1991 / Programme 1992 Erwin Stucki

1992/4 Sustainable Development in Rural Areas Some Methodological Issus

Urs Egger

1992/5 Landwirtschaftlicher Bodenmarkt und Bodenpolitik

Peter Rieder/Rolf Huber

1992/6 Abaisser les coûts de production ou comment maintenir notre avantage concurrentiel? Bernard Lehmann 
1992/8 Strategien für landwirtschaftliche Unternehmungen in der

Schweiz von morgen

Bernard Lehmann

1992/9 Introduction to Financial and Economic Analysis of Agri-

1992/10 LE SKI ALPIN: une industrie indispensable pour le develope-

1992/11 Espace, société et territoire une étude de cas: la commune de Bagnes

S. Guindani/N. Kessler/S. Gautschi

1992/12 Regionale und globale Umweltbelastungen durch die Landwirtschaft

Urs Egger

1992/13 Estimation monétaire des externalités de l'agriculture

(Identification des externalités de l'agriculture et étude des possibilitiés d'evaluation empirique)

Torsten Redies

1992/14 Stratégies pour entreprises agricoles suisses; und conception de base

Bernard Lehmann

1992/15 La Politique Agricole Commune: quelques repères

Dominique Barjolle

1993/1 Marketing-Organisation in der Schweizer Landwirtschaft

Robert Jörin

1993/2 Ideen und Geistesgeschichte der europäischen Agrarpolitik

1993/3 Eine wirtschaftswissenschaftliche Analyse der landwirtschaftlichen Nutzung organischer Böden in der Schweiz

Renan Goetz

1993/4 Agrarschutz für hors sol-Produkte?

Sibyl Anwander Phan-huy

1993/5 Lenkungsabgaben auf Dünger

Entscheidungselemente für die Einführung von Lenkungsabgaben auf Mineraldünger und Hofdüngerüberschüsse

Bernard Lehmann

1994/1 Setting Pigouvian Taxes Correctly - an Extension 
1994/3 Berglandwirtschaft in der Schweiz

1994/4 Commercialisation, Marketing agro-alimentaire

(exemples: produits régionaux)

B. Lehmann/D. Barjolle/ A. Silauri

1995/1 Colloque DGR/EPFL \& IER/ETHZ: inauguration Institut

d'économie rurale, antenne romande (IER-AR)

Erwin W. Stucki

1995/2 On tourism in Switzerland: tourism and sustainable

Erwin W. Stucki

1995/3 Transformation des modes d'exploitation dans les Alpes: contributions au Forum Alpin '94 de l'ASSN

Erwin W. Stucki

1995/4 Auswirkungen der Agrarpolitik 2002 auf die Schweizer

1995/5 Die Agrarstruktur- und Regionalpolitik der Europäischen

Union, eine Standortbestimmung

Daniel Löw

1995/6 Die Agrarstrukturpolitik Österreichs und deren Anpassung an die EU

Daniel Löw, Erwin Stucki

1995/7 Regionale Entwicklungsstrategien im ländlichen Raum Japans:

Fallstudie in Hayakawa-cho und Oguni-machi

Ch. Theler/M. Häfliger

1996/1 Swiss Agricultural Policy, the Swiss Agricultural Knowledge 


\section{$\underline{\text { Schriftenreihe/Publications }}$}

\section{BESTELLUNG/COMMANDE}

Name und Vorname/

Nom et prénom :

Adresse:

Schrift Nr./publication no

Einsenden an/

envoyer à :

Institut für Agrarwirtschaft,

zu Hd. Frau Lucia Keller

ETH-Zentrum

8092 ZÜRICH 